\title{
Transfer of Supramolecular Chirality in Block Copoly(thiophene)s
}

\author{
Karlien Van den Bergh, ${ }^{[a]}$ Jurgen Huybrechts, ${ }^{[b]}$ Thierry Verbiest, ${ }^{[a]}$ and Guy Koeckelberghs ${ }^{*[a]}$
}

Conjugated block copolymers have received considerable attention in recent years, as they hold promise, for instance for solar cells and nanoelectronics. ${ }^{[1-3]}$ Block copolymers consisting of one conjugated block and one or more nonconjugated, usually coil-like blocks, have been shown to possess a variety of defined morphologies which differ from those of the parent conjugated homopolymers. ${ }^{[1]}$ However, since the nonconjugated segments do not contribute electronically to the material, their role remains restricted to the self-assembly. In contrast, different morphologies and unique electronic properties can be expected for conjugated block copolymers composed of two, electronically different blocks. ${ }^{[2]}$ For instance, Tu et al. showed that conjugated block copolymers can form morphologies which differ from those of a blend of their homopolymers and that the morphology and corresponding optical properties of amphiphilic block copolymers depend on the solvent conditions. ${ }^{[3]}$ Here, we show that the electronic properties and the supramolecular organization of one conjugated block can also be influenced by the other conjugated block.

The block copolymer studied in this paper, is composed of a regioregular, head-to-tail coupled, (achiral) poly(3-hexylthiophene) (P3HT) and a chiral, regioregular, head-to-tail coupled poly(3-(3,7-dimethyloctyloxy)thiophene) (P3OOT). Although both polymers are composed of the same polythiophene backbone, they electronically differ significantly: for instance, $\lambda_{\max }$ in film varies from $\sim 500 \mathrm{~nm}$ for P3HT ${ }^{[4]}$ to $\sim 630 \mathrm{~nm}$ for P3OOT. ${ }^{[5]}$

Circular dichroism (CD) spectroscopy provides a very powerful tool in the study of the supramolecular organization of

[a] K. V. d. Bergh, T. Verbiest, G. Koeckelberghs Laboratory of Molecular Electronics and Photonics

Department of Chemistry

K.U.Leuven

Celestijnenlaan 200F, B-3001 Heverlee, Belgium

Fax: (+) 32 (0) 16327990

E-mail: guy.koeckelberghs@chem.kuleuven.be

[b] J. Huybrechts

Research group of Functional Genomics and Proteomics

Department of Biology

K.U.Leuven

Naamsestraat 52, B-3000 Leuven, Belgium

Supporting information for this article is available on the WWW under http://www.chemeurj.org/ or from the author.)) (chiral) conjugated polymers. Langeveld-Voss et al. have demonstrated that bisignate Cotton effects, commonly observed in films and poor solvent mixtures of regioregular polythiophenes, arise from chirally aggregated, coplanar polymer strands. ${ }^{[6]}$ Since these Cotton effects are located in the absorption band of the corresponding polymer, CD spectra can deliver ambiguous evidence for the presence of supramolecular chirality in the respective blocks of conjugated block copolymers, provided that both polymer blocks show a significantly different $\lambda_{\max }$. As a consequence, $\mathrm{CD}$ spectroscopy can probe a transfer of supramolecular chirality from the chiral P3OOT to the achiral P3HT block, as this would induce a bisignate Cotton effect in the absorption band of the achiral P3HT block.

The block copolymer was prepared by successive addition of the alkyl-substituted and alkoxy-substituted thiophene monomers, taking advantage of the living character of the Ni-catalyzed polymerization of $\mathbf{2}$ (Scheme 1) ${ }^{[7]}$ Special care was devoted to the regioregularity of the resulting polymer blocks, since this is a requisite for efficient supramolecular stacking, which was accomplished by the use of reported polymerization methods. ${ }^{[8]}$ For the P3OOT block, this requires the exclusive formation of $\mathbf{5}$, being the most stable monomeric isomer. ${ }^{[6]}$ Since the polymerization of $\mathbf{2}$ in the presence of $\mathrm{Ni}(\mathrm{dppp}) \mathrm{Cl}_{2}(\mathrm{dppp}=1,3-$ bis(diphenylphosphino)propane) has been shown to proceed via a living chain-growth mechanism, ${ }^{[7 a, 9]}$ while it is uncertain whether this is also the case for alkoxy-substituted thiophenes, ${ }^{[10]}$ first the P3HT block was formed, from which the P3OOT block was grown. The ratio $[2] /\left[\mathrm{Ni}(\mathrm{dppp}) \mathrm{Cl}_{2}\right]=15$. In order to verify the living nature of the P3HT block at the moment the second monomer was introduced and to characterize the P3HT block formed, the reaction mixture was divided in two parts. A first aliquot was quenched with $\mathrm{HCl}$ (1 $\mathrm{M}$ in methanol), rendering P3HT $_{1}$. 5 was added to the second batch. The actual concentration of $\mathbf{5}$ was determined by quenching a small sample of the reaction mixture with $\mathrm{D}_{2} \mathrm{O}$ and analyzing it with ${ }^{1} \mathrm{H}$ NMR spectroscopy. This revealed that $\mathbf{5}$ was formed in $50 \%$ (the remainder being 4), which corresponds to $[\mathbf{5}] /[\mathbf{3}]=23$. After polymerization, also the second batch was quenched with $\mathrm{HCl}$ (1 $\mathrm{M}$ in methanol), rendering P3HT-b-P3OOT.

The polymers were subjected to MALDI-TOF mass spectrometry and GPC. According to MALDI-TOF, the degree of polymerization $\left(\mathrm{P}_{\mathrm{n}}\right)$ of $\mathbf{P 3 H \mathbf { T } _ { 1 }}$ amounts 9.5, which corresponds to a conversion of $\sim 63 \%$; GPC analysis resulted in somewhat higher values $\left(M_{n}=2,8 \mathrm{~kg} / \mathrm{mol}\right)$. The polymer solely consists of $\mathrm{H} / \mathrm{Br}$ 
end-groups, which is expected for the living polymerization of 2. ${ }^{[7 a, 9]}$ Unfortunately, we failed in analyzing P3HT- $\boldsymbol{b}$-P3OOT using MALDI-TOF spectrometry. Nevertheless, the absence of P3HT $_{1}$ in the GPC profile of P3HT- $b$-P3OOT demonstrates that all $\mathbf{3}$ initiated the polymerization of $\mathbf{5}$ and its unimodal GPC profile at smaller elution volumes delivers a final proof of the living nature of 3 . Moreover, the fact that the peak strongly absorbs at both $430 \mathrm{~nm}$, at which P3HT strongly absorbs but P3OOT less, and at $600 \mathrm{~nm}$, at which P3OOT absorbs but P3HT not, confirms that it must originate from a block copolymer composed of both a P3HT and a P3OOT block. Fortunately, $\mathrm{P}_{\mathrm{n}}$ of P3HT-b-P3OOT could conveniently be calculated from its ${ }^{1} \mathrm{H}$ NMR spectrum by relative integration of signals arising from the P3HT block (9.5 units) and the P3OOT block, which revealed that the P3OOT block has $\mathrm{P}_{\mathrm{n}}$ of $\sim 14$.

In order to investigate whether the polymer blocks influence their mutual supramolecular organization, the solvent quality of a solution of P3HT-b-P3OOT was systematically decreased, which was accomplished by gradual addition of methanol to a chloroform solution, and the influence on the UV/Vis and CD spectra was evaluated. In a chloroform solution, P3HT is present as poorly conjugated coils, while $\mathrm{P} 3 \mathrm{OOT}$ rather adopts a strongly conjugated, planar conformation. ${ }^{[1]}$ Importantly, the UV/Vis spectrum of the block copolymer is not a superposition of the constituting homopolymers; it rather corresponds to a superposition of P3OOT and a P3HT of higher $\mathrm{P}_{\mathrm{n}}$. This demonstrates that both conjugated blocks do influence each other electronically.

At $30 \%$ nonsolvent content, the P3OOT block is aggregated, which is evidenced by the appearance of bisignate Cotton effects in the absorption band of the P3OOT block (Figure 1a-b). Since P3OOT is already strongly conjugated in chloroform solution, its aggregation is accompanied by a limited gain in conjugation and moderate red-shift $(\sim 30 \mathrm{~nm})$, resulting in only a slight change of the UV/Vis spectrum. At this point, the P3HT block is still present in its unaggregated, poorly conjugated conformation. However, at $60 \%$ methanol, also the P3HT block is aggregated, as evidenced by the UV/Vis spectra, which evolve to a combination of P3OOT and P3HT blocks, both in their planar and aggregated state. However, the spectra are no superposition of their constituting homopolymers, demonstrating the mutual electronic influence of the two blocks.

Importantly, the zero-crossing of the CD spectrum displays a blue-shift (from $\sim 600$ to $\sim 550 \mathrm{~nm}$ ) and its intensity almost triples (from $\mathrm{g}_{\mathrm{abs}, \lambda=550 \mathrm{~nm}}=-2 \cdot 10^{-3}$ to $\mathrm{g}_{\mathrm{abs}, \lambda=480 \mathrm{~nm}}=-10^{-2}$ ). In fact, it is a superposition of two bisignate Cotton effects - one near $600 \mathrm{~nm}$ and one near $500 \mathrm{~nm}$ - originating from, respectively, chirally aggregated P3OOT and P3HT. Therefore, this experiment indicates that the chiral supramolecular organization of P3OOT invokes supramolecular chirality in the achiral P3HT block.

Next, the same experiment was repeated with blends of P3OOT $\left(\mathrm{M}_{\mathrm{n}}=22 \mathrm{~kg} / \mathrm{mol}, \mathrm{GPC}\right)^{[12]}$ and P3HTs of different molar masses. Blends, composed of P3HT $\mathbf{P}_{2}\left(\mathbf{M}_{\mathrm{n}}=2,4 \mathrm{~kg} / \mathrm{mol}\right.$, GPC) which is similar to the P3HT block, could not be used, since the methanol content required to induce aggregation exceeds the highest attainable content with the protocol used. P3HT $\mathbf{T}_{3}\left(\mathbf{M}_{\mathrm{n}}=\right.$ $4,1 \mathrm{~kg} / \mathrm{mol}, \mathrm{GPC})$, on the other hand, better resembles the P3HT block of P3HT- $\boldsymbol{b}$-P3OOT both electronically and in the methanol content required to induce aggregation. At 30\% methanol, P3OOT chirally stacks, while $\mathbf{P 3 H T}_{3}$ is still present as unaggregated, random coils. At $60 \%$ methanol, also $\mathbf{P 3 H T}_{3}$ aggregates, as evidenced by the UV/Vis spectra. On the other hand, the CD spectra do not gain intensity $\left(\mathrm{g}_{\mathrm{abs}, \lambda} \lambda=550 \mathrm{~nm}=-4 \cdot 10^{-3}\right)$ and the zero-crossing displays a small blue-shift which is similar to that of P3OOT but clearly different from the block copolymer, showing that a contribution of $\mathbf{P 3 H T}_{\mathbf{3}}$ is absent. As a consequence, it must be concluded that the two polymers separately aggregate and do not influence their mutual supramolecular organization in the blend, but that transfer of supramolecular chirality is present in the block copolymer.

It is clear that blends of $\mathbf{P B H T}_{\mathbf{3}}$ and P3OOT form separate aggregates and that chirality is absent in the $\mathrm{P} 3 \mathrm{HT}$ aggregates because no chiral P3OOT chains are incorporated in the P3HT aggregates. However, Langeveld-Voss et al. demonstrated that blending achiral and chiral poly(3-alkylthiophene)s which aggregate at the same methanol content, results in mixed aggregates in which a sergeant-and-soldiers behavior is present. ${ }^{[13]}$ To investigate whether this is also the case for P3HTP3OOT blends or that both polymers inherently segregate, the aggregation of P3HT $_{4}\left(\mathrm{M}_{\mathrm{n}}=4.1 \mathrm{~kg} / \mathrm{mol}, \mathrm{GPC}\right)$ and P3OOT, which aggregate at the same methanol content, was studied. The $\mathrm{CD}$ spectra, in particular the zero-crossing around $550 \mathrm{~nm}$, indicate that both polymers are chirally stacked. Therefore, it can be concluded that the aggregates contain both achiral $\mathbf{P 3 H T}_{\mathbf{4}}$ and chiral P3OOT chains. On the other hand, the intensity is somewhat lower $\left(\mathrm{g}_{\mathrm{abs}, \lambda}=550 \mathrm{~nm}=-1.5 \cdot 10^{-3}\right)$, which may point at a more complicated stacking if the aggregates are composed of chains of a different nature. Importantly, the different behavior of the P3HT $_{3}$-P3OOT and P3HT $_{4}$-P3OOT blends again confirms the unique supramolecular behavior of the block copolymer.

In a next experiment, films of P3HT-b-P3OOT were spincoated. The samples were annealed by heating them at progressively increasing temperatures for $1 \mathrm{~min}$. After each heating cycle, the samples were quenched to room temperature and their UV/Vis and CD spectra were immediately recorded. At $110{ }^{\circ} \mathrm{C}$, the UV/Vis and CD spectra resemble well those in a poor solvent mixture, indicating that both polymer blocks are chirally aggregated. At higher temperatures, the contribution of the P3HT block vanishes, which can be associated with the melting of the P3HT block.

Then, a film, annealed at $110^{\circ} \mathrm{C}$, was oxidized by exposure to $\mathrm{I}_{2}$ vapor (Figure 2). Although the $\mathrm{CD}$ spectrum of the oxidized block copolymer is less defined, it is clear that chirality is still present $\left(\mathrm{g}_{\mathrm{abs}}=-10^{-1} ; \lambda=460\right.$ and $700 \mathrm{~nm}$ ). Interestingly, $\mathrm{I}_{2}$-doped polythiophenes tend to de-dope - the rate of reduction being dependent on the electronic nature of the polythiophene. Indeed, after 5 weeks the original spectra are restored, indicating that the supramolecular chiral organization is preserved during oxidation and back reduction. A similar behavior has also been found for chiral poly(dithienopyrrole) $\mathrm{s}^{[14]}$ and $\operatorname{poly}(3,4-$ dialkoxythiophene)s ${ }^{[15]}$. Since P3HT is much faster dedoped then P3OOT, the P3HT block is first reduced, followed by the P3OOT block. Unfortunately, since the UV/Vis and CD spectra of P3HT$\boldsymbol{b}$-P3OOT are not a simple superposition of contributions of the two blocks, the individual contributions could not be calculated. ${ }^{[16]}$. Nevertheless, it is clear that the spectra of P3HT- $b$ P3OOT after 4 days are a combination of oxidized (chiral) 
P3OOT and neutral, chirally aggregated P3HT. This again demonstrates that the achiral P3HT block shows supramolecular chirality, imposed by the chiral P3OOT block.

In conclusion, we have prepared a block copolymer, composed of (achiral) P3HT and chiral P3OOT and demonstrated that the P3OOT block influences the supramolecular organization of the P3HT block, as is expressed by transfer of supramolecular chirality to the achiral P3HT block. Further research will focus on the morphology of films of the block copolymer.

\section{Experimental Section}

Synthesis of P3HT $_{1}$ and P3HT- $\boldsymbol{b}$-P3OOT.

A solution of $1(0.50 \mathrm{mmol}, 0.186 \mathrm{~g})$ in dry THF $(2.5 \mathrm{~mL})$ was cooled to $0{ }^{\circ} \mathrm{C}$ and $i \mathrm{PrMgCl}(0.50 \mathrm{mmol}, 0.25 \mathrm{~mL}, 2 \mathrm{M}$ in THF) was added. The reaction mixture was stirred for $1 \mathrm{~h}$ at $0{ }^{\circ} \mathrm{C}$ and then cannulated to a suspension of $\mathrm{Ni}(\mathrm{dppp}) \mathrm{Cl}_{2}(33.1 \mu \mathrm{mol}$, $17.5 \mathrm{mg})$ in dry THF $(3.5 \mathrm{~mL})$. The volume of the polymerization reaction was adjusted to $7 \mathrm{~mL}$. After stirring for $2 \mathrm{~h}$, a small aliquot $(1.4 \mathrm{~mL})$ was withdrawn and quenched with $\mathrm{HCl}$ (1 M in methanol) (P3HT 1 ). 5 ( $\sim 0.60 \mathrm{mmol}$ in THF) was added to the remaining part and the reaction mixture was stirred overnight. After being quenched with $\mathrm{HCl}(1 \mathrm{M}$ in methanol), the polymer was precipitated into methanol, filtered off and dried (P3HT-b-P3OOT). For the preparation of 5, freshly prepared lithium diisopropylamide ( $1.32 \mathrm{mmol}$ in $3 \mathrm{~mL}$ of THF) was added to a solution of 4 $(1.20 \mathrm{mmol}, 0.383 \mathrm{~g})$ in dry THF $(3 \mathrm{~mL})$ at $-78^{\circ} \mathrm{C}$. After stirring for $30 \mathrm{~min}$ at RT, the solution was added to a suspension of $\mathrm{MgBr}_{2}(1.80 \mathrm{mmol}, 0.331 \mathrm{~g})$ in dry THF $(4 \mathrm{~mL})$ and stirred for another $30 \mathrm{~min}$.

The homopolymers $\left(\right.$ P3OOT $^{[5]}$, P3HT $_{2-4}{ }^{[9]}$ ) were prepared according to literature procedures.

\section{Acknowledgements}

This work was supported by the Katholieke Universiteit Leuven (GOA) and the Fund for Scientific Research (FWO-Vlaanderen). K. V. d. B. is a doctoral fellow of the IWT, G. K. and J. H. are postdoctoral fellows of FWO-Vlaanderen.

Keywords: chirality $\cdot$ block copolymers $\cdot$ supramolecular chemistry $\cdot$ conjugated polymers

[1] For a selection of papers: a) G. Widawski, M. Rawiso, B. François, Nature 1994, 369, 387; b) S. A. Jenekhe, X. L. Chen, Science 1998, 279, 1903; c) M. A. Hempenius, B. M. W. Langeveld-Voss, J. A. E. H. van Haare, R. A. J. Janssen, S. S. Sheiko, J. P. Spatz, M. Möller, E. W. Meijer, J. Am. Chem. Soc. 1998, 120, 2798; d) G. N. Tew, M. U. Pralle, S. I. Stupp, J. Am. Chem. Soc. 1999, 121, 9852; e) W. Li, H. Wang, L. Yu, T. L. Morkved, H. M. Jaeger, Macromolecules 1999, 32, 3034; f) U. Stalmach, B. de Boer, C. Videlot, P. F. van Hutten, G. Hadziioannou, J. Am. Chem. Soc. 2000, 122, 5464; g) H. Wang, H. H. Wang, V. S. Urban, K. C. Littrell, P. Thiyagarajan, L. Yu, J. Am. Chem. Soc. 2000, 122, 6855; h) P. Leclère, E. Hennebicq, A. Calderone, P. Brocorens, A. C. Grimsdale, K. Müllen, J.-L. Brédas, R. Lazzaroni, Prog.
Polym. Sci. 2003, 28, 55; i) J. Liu, E. Sheina, T. Kowalewski, R. D McCullough, Angew. Chem. Int. Ed. 2002, 41, 329; Angew. Chem. 2002, 114, 339.

[2] For a selection of papers: a) C. Schmitt, H. G. Nothofer, A. Falcou, U. Scherf, Macromol. Rapid Commun. 2001, 22, 624; b) H. Wang, M. K. Ng, L. Wang, L. Yu, B. Lin, M. Meron, Y. Xiao, Chem. Eur. J. 2002, 8, 3246; c) M. K. Ng, L. Yu, Angew. Chem. Int. Ed. 2002, 41, 3598; Angew. Chem. 2002, 114 3750; d) U. Asawapirom, R. Güntner, M. Forster, U. Scherf, Thin Solid Films $\mathbf{2 0 0 5}, 477,48$.

[3] a) G. Tu, H. Li, M. Forster, R. Heiderhoff, L. J. Balk, U. Scherf, Macromolecules 2006, 39, 4327; b) G. Tu, H. Li, M. Forster, R. Heiderhoff, L. J. Balk, R. Sigel, U. Scherf, Small 2007, 3, 1001.

[4] R. D. McCullough, R. L. Lowe, M. Jayaraman, D. L. Anderson, J. Org. Chem. 1993, 58, 904.

[5] G. Koeckelberghs, M. Vangheluwe, C. Samyn, A. Persoons, T. Verbiest, Macromolecules 2005, 38, 5554.

[6] B. M. W. Langeveld-Voss, D. Beljonne, Z. Shuai, R. A. J. Janssen, S. C. J. Meskers, E. W. Meijer, J.-L. Brédas, Adv. Mater. 1998, 10, 1343.

[7] Subsequent addition of different alkyl-substituted thiophene monomers has been applied to prepare block copoly(3-alkyl-thiophene)s: a) M. C. Iovu, E. E. Sheina, R. R. Gil, R. D. McCullough, Macromolecules 2005, 38, 8649; b) T. Yokozawa, I. Adachi, R. Miyakoshi, A. Yokoyama, High Perform Polym 2007, 19, 684 .

[8] R. S. Loewe, P. C. Ewbank, J. Liu, L. Zhai, R. D. McCullough, Macromolecules 2001, 34, 4324.

[9] R. Miyakoshi, A. Yokoyama, T. Yokozawa, J. Am. Chem. Soc. 2005, 127, 17542 .

[10] A manuscript concerning the polymerization of poly(3-alkoxythiophene)s is in preparation.

[11] M. Vangheluwe, T. Verbiest, G. Koeckelberghs, Macromolecules 2008, 41, 1041 .

[12] Due to the rigid, rod-like conformation of poly(3-alkoxythiophene)s, a large overestimation of the molar mass using GPC toward polystyrene standards can be expected. MALDI-TOF spectroscopy on P3OOT resulted in $\mathrm{M}_{n}=5$ $\mathrm{kg} / \mathrm{mol}, \mathrm{P}_{\mathrm{n}}=20$, which corresponds rather well to the P3OOT block.

[13] B. M. W. Langeveld-Voss, R. J. M. Waterval, R. A. J. Janssen, E. W. Meijer, Macromolecules 1999, 32, 227.

[14] G. Koeckelberghs, L. De Cremer, W. Vanormelingen, T. Verbiest, A. Persoons, C. Samyn, Macromolecules 2005, 38, 4545.

[15] C. R. G. Grenier, S. J. George, T. J. Joncheray, E. W. Meijer, J. R. Reynolds, J. Am. Chem. Soc. 2007, 129, 10694.

[16] Spectra of oxidized P3OOT are presented in Supporting Information.

Received: ((will be filled in by the editorial staff)) Revised: ((will be filled in by the editorial staff)) Published online: ((will be filled in by the editorial staff)) 


\section{Captions to scheme and figures}

Scheme 1. Synthesis of the homo- and block copolymer.

Figure 1. UV/Vis and CD spectra of P3HT-b-P3OOT (a, b), a blend of P3HT $\mathbf{3}(\mathrm{c}=74 \mathrm{mg} / \mathrm{L})$ and P3OOT $(\mathrm{c}=40 \mathrm{mg} / \mathrm{L})(\mathrm{c}, \mathrm{d})$ and a blend of P3HT $4(\mathrm{c}=29 \mathrm{mg} / \mathrm{L})$ and P3OOT $(\mathrm{c}=24 \mathrm{mg} / \mathrm{L})(\mathrm{e}, \mathrm{f})$. (- $0 \%$ methanol,---- $30 \%$ methanol, $\cdots \cdots 60 \%$ methanol).

Figure 2. UV/Vis and CD spectra of the oxidation $\left(\mathrm{I}_{2}\right)$ and back reduction of P3HT- $\boldsymbol{b}$-P3OOT (before oxidation,--- after oxidation, $\cdots$ after 4 days, ---. after 5 weeks). 


\section{Entry for the Table of Contents}

\section{Block-copolymers}

Karlien Van den Bergh, Jurgen Huybrechts, Thierry Verbiest, Guy Koeckelberghs* Page-Page

\section{Transfer of Supramolecular}

\section{Chirality in Block}

\section{Copoly(thiophene)s}

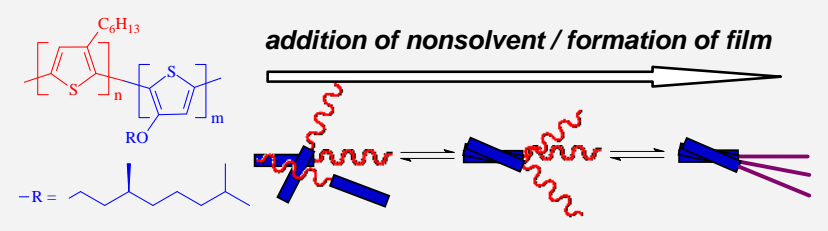

A block copolymer, composed of an chiral block transfers its (achiral) poly(3-hexylthiophene) and a chiral, poly (3- $(3,7-$ dimethyloctyloxy)thiophene) was prepared. It was shown that the supramolecular chirality to the achiral block, demonstrating that the blocks influence their mutual supramolecular organization. 